\title{
Impact of the COVID-19 pandemic on the behaviour and health status of patients with COPD: results from the German COPD cohort COSYCONET
}

\author{
Kathrin Kahnert ${ }^{1}$, Johanna I. Lutter ${ }^{2}$, Tobias Welte $\mathbb{C}^{3}$, Peter Alter $\mathbb{1}^{4}$, Jürgen Behr ${ }^{1}$, Felix Herth $\mathbb{C}^{5}$, \\ Hans-Ulrich Kauczor ${ }^{6}$, Sandra Söhler ${ }^{4}$, Michael Pfeifer ${ }^{7}$, Henrik Watz ${ }^{8}$, Claus F. Vogelmeier ${ }^{4}$, Robert Bals $\mathbb{C}^{9}$, \\ Rudolf A. Jörres ${ }^{10}$ and Franziska C. Trudzinski $\mathbb{D}^{5}$
}

\begin{abstract}
${ }^{1}$ Dept of Medicine V, University Hospital, Ludwig-Maximilians-University (LMU) Munich, Comprehensive Pneumology Center Munich (CPC-M), German Center for Lung Research (DZL), Munich, Germany. ${ }^{2}$ Institute of Health Economics and Health Care Management, Helmholtz Zentrum München $\mathrm{GmbH}$ - German Research Center for Environmental Health, CPC-M, DZL, Munich, Germany. ${ }^{3}$ Dept of Pneumology, Hannover Medical School, Hannover, Germany. ${ }^{4}$ Dept of Medicine, Pulmonary and Critical Care Medicine, Philipps University of Marburg (UMR), DZL, Marburg, Germany. ${ }^{5}$ Dept of Pneumology and Critical Care Medicine, Thoraxklinik University of Heidelberg, Translational Lung Research Center Heidelberg (TLRC-H), DZL, Heidelberg, Germany. ${ }^{6}$ Dept of Diagnostic and Interventional Radiology, University Hospital of Heidelberg, DZL, Heidelberg, Germany. ${ }^{7}$ Dept of Respiratory Medicine, Donaustauf Hospital, Regensburg, Germany. ${ }^{8}$ Pulmonary Research Institute at LungenClinic Grosshansdorf, Airway Research Center North (ARCN), DZL, Grosshansdorf, Germany. ${ }^{9}$ Dept of Internal Medicine V - Pulmonology, Allergology, Critical Care Care Medicine, Saarland University Hospital, Homburg, Germany. ${ }^{10}$ Institute and Outpatient Clinic for Occupational, Social and Environmental Medicine, LMU, LMU University Hospital, CPC-M, DZL, Munich, Germany.
\end{abstract}

Corresponding author: Kathrin Kahnert (kathrin.kahnert@med.uni-muenchen.de)

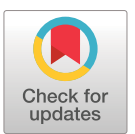

Copyright OThe authors 2021

This version is distributed under the terms of the Creative Commons Attribution NonCommercial Licence 4.0. For commercial reproduction rights and permissions contact permissions@ersnet.org

This article has supplementary material available from openres.ersjournals.com

Received: 8 April 2021 Accepted: 8 June 2021

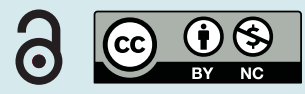

Shareable abstract (@ERSpublications)

During the period of \#COVID19 restrictions, slight deteriorations of clinical state with increasing adherence to protective measures were observed. In particular, women and GOLD D patients are at risk of deterioration. https://bit.ly/2S7fhEo

Cite this article as: Kahnert K, Lutter JI, Welte T, et al. Impact of the COVID-19 pandemic on the behaviour and health status of patients with COPD: results from the German COPD cohort COSYCONET. ERJ Open Res 2021; 7: 00242-2021 [DOI: 10.1183/23120541.00242-2021].

\section{Abstract}

Background: Infection control measures for coronavirus disease 2019 (COVID-19) might have affected management and clinical state of patients with COPD. We analysed to which extent this common notion is fact-based.

Methods: Patients of the COSYCONET cohort were contacted with three recurring surveys (COVID1, 2 and 3 at 0,3 and 6 months, respectively). The questionnaires comprised behaviour, clinical and functional state, and medical treatment. The responses to the questionnaires were compared amongst themselves and with pre-COVID information from the last visit of COSYCONET.

Results: Overall, 594 patients were contacted and 375 patients (58\% males, forced expiratory volume in $1 \mathrm{~s}\left(\mathrm{FEV}_{1}\right) 61 \pm 22 \%$ predicted) provided valid data in COVID1 and COVID2. Five patients reported infections with severe acute respiratory syndrome coronavirus 2 (SARS-CoV-2). Most patients - except for patients with higher education - reported compliance with recommended protective measures, whereby compliance to hygiene, contact and access to physicians slightly improved between COVID1 and COVID2. Also, patients obtained more information from physicians than from public media. In the majority of cases, the personal physician could not be substituted by remote consultation. Over time, symptoms slightly increased and self-assessed physical capacity decreased. Results of COVID3 were similar. Women and patients with more exacerbations and dyspnoea avoided medical consultations, whereas Global Initiative for Chronic Obstructive Lung Disease (GOLD) D patients were more amenable to tele-consultation.

Conclusion: In well-characterised COPD patients, we observed on average slight deteriorations of clinical state during the period of COVID-19 restrictions, with high and partially increasing adherence to protective measures. The data suggest that in particular, women and GOLD D patients should be actively contacted by physicians to identify deteriorations. 
Introduction

On March 11, 2020, coronavirus disease 2019 (COVID-19) was declared a pandemic and on March 22, 2020 Germany declared its first lockdown. Even though no general curfew was enacted, restrictions regarding personal contacts were imposed. While treating COVID-19-positive patients and tracing relevant contacts, the public health system tried to uphold medical care as much as possible. Patients with COPD are considered a risk group for severe COVID-19 courses [1, 2]. At the same time, these patients regularly require care from specialised clinics or practices. Therefore, public infection control measures can not only have beneficial effects, but also adverse effects on COPD patients, especially when access to medical care, physiotherapy and pulmonary exercise groups is limited by such public measures [3, 4].

A cross-sectional investigation of the experiences of COPD patients during Spain's first lockdown indicated only a low impact of disease control measures on their disease [4]. However, with the prolongation and tightening of such measures as the pandemic progressed, effects on the disease status may have become more pronounced over time. A detailed analysis of long-term changes and their impact on individual clinical history is not available yet but may be useful to improve the management of COPD patients in a pandemic.

The aim of the present study was to monitor the impact of the COVID-19 pandemic on COPD patients through repeated surveys. The focus of the surveys was placed on patients' behaviour, potential limitations of medical treatment and burdens of the disease, including depression scores. The study was conducted within the COSYCONET framework (COPD and Systemic Consequences-Comorbidities Network) between May 2020 and February 2021 and comprised three consecutive assessments. The relation to COSYCONET offered the opportunity to link questionnaire data to individual pre-COVID information on lung function, comorbidity profile and psychological status.

\section{Methods}

Study population

Patients of the German COPD cohort COSYCONET, which is a multi-centre study focusing on the role of comorbidities in COPD [5], and participating at visit 6 (6 years after recruitment) were contacted by mail and asked to respond to a specifically designed COVID-19 questionnaire (see supplementary material). The COVID-19 questionnaire included questions to capture patient behaviours such as hygiene measures, disease-specific behaviour, physician and physical therapy visits, use of video and telephone consultations, health status and physical activity. Details on COSYCONET, its inclusion/exclusion criteria and assessments have been published previously [5]. Fifteen study centres participated in this sub-study, whereas five could not participate due to logistic reasons during the pandemic. The COVID-19 questionnaire was at three intervals (at 0,3 and 6 months). The mailing of the COVID-19 questionnaire started from May to July 2020, August to October 2020, and November 2020 to January 2021 for the assessments scheduled at 0,3 and 6 months, respectively. After return of the questionnaires, the data base was finalised in February 2021. The collection of data over a 6-month period aimed to map potential long-term behavioural and health status-related changes during the evolving pandemic. The three consecutive questionnaires are referred to as COVID1, COVID2 and COVID3. The primary analysis focused on patients who returned at least COVID1 and COVID2, as the number of participants dropped from 564 in the first to 381 in the second to 241 in the third iteration of the survey; the latter is only shown in a secondary analysis.

The COSYCONET study has been approved by the ethical committees of all study centres, and all patients gave their written informed consent [5]. The additional examination of patients with the COVID questionnaire was also approved by the Ethics Committee (COSYCONET study, Amendment 11, AZ 200/09). The COSYCONET study was conducted in accordance with the Declaration of Helsinki.

\section{Assessments}

During the regular visits of the COSYCONET study, a detailed recording of all COPD-specific characteristics, demographics, comorbidities, medication, generic and disease-specific quality of life, and psychological disorders was performed [5]. Lung function was assessed following the standard operating procedures of COSYCONET in accordance with guidelines [6, 7]. The assessments included forced expiratory volume in $1 \mathrm{~s}\left(\mathrm{FEV}_{1}\right)$ and its ratio $\left(\mathrm{FEV}_{1} / \mathrm{FVC}\right)$ to forced vital capacity (FVC), as well as diffusing capacity of the lung for carbon monoxide $(\mathrm{CO})$ in terms of transfer factor $\left(T_{\mathrm{LCO}}\right)$ and transfer coefficient of the lung for carbon monoxide $\left(K_{\mathrm{CO}}\right)$. Predicted values for spirometry and diffusing capacity were taken from the Global Lung Function Initiative (GLI) $[8,9]$. On the basis of the lung function at the time of recruitment, we also determined the annual decline of lung function over the follow-up visits prior 
to the COVID-19 pandemic, to be able to assess the potential link between the responses to the questionnaire and the decline in lung function.

Symptom burden was evaluated by using the disease-specific COPD Assessment Test (CAT) and the Modified Medical Research Council (mMRC) dyspnoea scale [10, 11]. The Patient Health Questionnaire Depression Scale 9 (PHQ-9) was used as a tool for screening depression [12]. Education was categorised in three groups based on the number of years of education completed (basic education $\leqslant 9$ years, secondary education 10-11 years, higher education $>11$ years) [13]. We used data of the COSYCONET visit 6 for comparison with pre-pandemic measurements. The median time interval between visit 6 and the first COVID survey was 1.6 years.

\section{COVID-19 restrictions in Germany}

In Germany, the first lockdown was declared on March 22, 2020. The restrictions included control measures such as social distancing and limitations of personal contacts, and accordingly hospitality, recreational and cultural facilities were shut. These restrictions were attenuated between May and October 2020 on a local basis, due to the decrease in the daily number of cases. From April 29, 2020 onwards, the wearing of face masks had become compulsory in public transport sytstems, schools and shops. During the first lockdown, non-urgent physician visits in both outpatient and inpatient settings were cancelled by the healthcare providers, postponed or replaced by telephone visits. In the time between May and November 2020, the German healthcare system's capacity was at no time exhausted, and all healthcare services could be offered to the fullest extent. The second wave of the pandemic led to a further lockdown (called "Lockdown Light”), which was declared on November 2, 2020. Several cultural and business institutions were shut down again, but the school system was initially kept open. These measures were tightened on December 13, 2020 and were still in place at the end of our survey. However, the second lockdown did not lead to a general reduction in healthcare services, and elective surgical procedures were postponed only in individual hot-spot areas owing to increased utilisation of intensive care units.

\section{Statistical analyses}

Regarding baseline characteristics of the study population and dropouts, differences between groups were identified using chi-square tests for categorical variables and analysis of variance (ANOVA) for continuous variables. To analyse changes in CAT, PHQ-9 and mMRC scores prior to versus during the pandemic and over the course of the pandemic, mean values were compared using pairwise t-tests. Additionally, the PHQ-9 categories "healthy to severe depression" [12] were compared using Wilcoxon signed-rank tests for dependent samples.

The questions in the COVID questionnaire had to be answered on a 5-item scale with the response options $1=$ "does not apply at all” to 5="fully applies”. Differences in the distribution of responses between COVID1 and COVID2 were analysed by histograms and Wilcoxon signed-rank test for dependent samples. To analyse the responses from COVID1 to COVID 3, the nonparametric Friedman test for repeated measures was used. Furthermore, we investigated the dependence of responses in COVID1 on several patient characteristics using chi-square tests. Statistical analyses were performed using SAS software (version 9.4; SAS Institute Inc., Cary, NC, USA), and p-values of $\leqslant 0.05$ were considered statistically significant.

\section{Results}

\section{Study population}

Overall, 594 patients from 15 study centres were contacted, of whom 565 (95\%) responded to at least one questionnaire. In total, we received 1186 complete COVID-19 questionnaires, 565 COVID1, 381 COVID2 and 241 COVID3. Complete responses to COVID 1 and COVID 2 were available for 375 patients, and 223 patients returned all three questionnaires. For purposes of the primary analysis, we defined the 375 patients as study population who returned both the COVID1 and COVID2 questionnaire (see figure 1, consort diagram). Five out of all 565 patients reported to have been diagnosed with COVID-19.

Table 1 shows patients' characteristics obtained at the COSYCONET visit 6, i.e. prior to COVID, and stratified according to the study population and the excluded patients. Significant differences between groups were found for the frequencies of gastrointestinal disorders, asthma and treatment with oral corticosteroids $(p<0.05$, each), whereas the distribution of sex, age, Global Initiative for Chronic Obstructive Lung Disease (GOLD) grades and groups, and educational status was not different. 


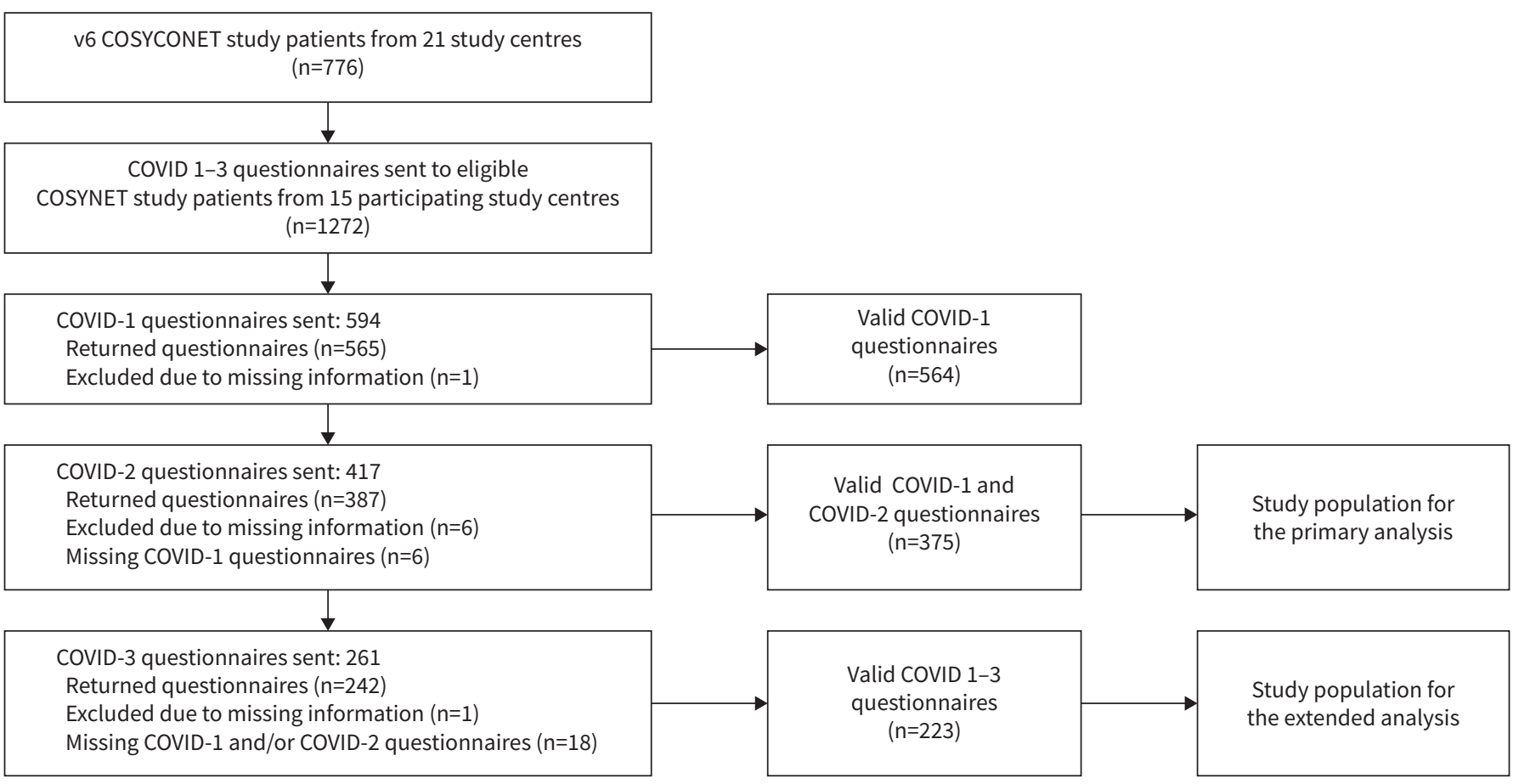

FIGURE 1 CONSORT flow chart of coronavirus disease 2019 (COVID-19) survey study participants. The questionnaires were only sent out again by the study centres if the first or second questionnaire was returned. Furthermore, owing to the increasing capacity bottlenecks caused by the COVID-19 pandemic, not all study centres were able to send out the questionnaires at the third timepoint. This led to differences in the number of questionnaires sent out on each occasion.

\section{Results of the repeated surveys}

The comparison of COVID1 and COVID2 revealed a small increase in total CAT score $(\mathrm{p}<0.05)$, specifically CAT item 3 (chest tightness). The increase in mMRC score was not statistically significant. For PHQ-9, COVID2 showed a higher proportion of the upper three categories, due to an increase in mild depression $(\mathrm{p}<0.05$; table 2$)$.

\section{Individual measures of hygiene}

Figure 2a shows the results regarding individual measures of hygiene, i.e. hand disinfection, use of face masks, social distancing and dispensing with travels. The only apparent change between COVID1 and COVID2 was the higher adherence to hand disinfection in the second questionnaire; there was an increase of $7 \%$ in the answer "fully applies" for "regular hand disinfection".

\section{Disease-specific behaviour}

Figure 2b shows the results relating to the avoidance of physicians' practices, physiotherapy, breathing therapy and pulmonary exercise groups. Interestingly, the level of avoidance of physicians' practices decreased in COVID2. While $16 \%$ of patients gave their full agreement for "avoidance of contact to practices” in COVID1, only 10\% of patients gave this answer in COVID2.

\section{Access to practices}

Figure 2c shows the results regarding the availability of physicians and specialists as well as the possibility to arrange for appointments at practices. Most patients reported a high accessibility to general practitioners and pulmonologists. Only a minority of patients and practices cancelled scheduled visits, and the number of cancelled visits (answer "fully applies") decreased from 13\% in COVID1 to 8\% in COVID2.

\section{Video or telephone consultation}

Figure $2 \mathrm{~d}$ shows the results regarding the frequency of tele-consultation, the attitude towards such consultation and its estimated effectiveness. The majority of patients did not use tele-consultation (68\% in COVID1 and 74\% in COVID2), did not consider tele-consultation as a suitable substitute for consultation in person (69\% in COVID1 and 75\% in COVID2) and did not, generally, favour tele-consultation (88\% in COVID1 and 91\% in COVID2). 


\begin{tabular}{|c|c|c|c|}
\hline & Study population & Excluded & $\mathrm{p}$-value \\
\hline Subjects n & 375 & 189 & \\
\hline Male & $218(58.1)$ & $109(57.7)$ & 0.9165 \\
\hline Age years & $71.1 \pm 8.4$ & $70.4 \pm 8.7$ & 0.3737 \\
\hline \multicolumn{4}{|l|}{ Education } \\
\hline Basic & $188(50.1)$ & $76(40.2)$ & 0.0829 \\
\hline Secondary & $117(31.2)$ & $70(37.0)$ & \\
\hline Higher & $70(18.7)$ & $43(22.8)$ & \\
\hline \multicolumn{4}{|l|}{ Smoking status } \\
\hline Current smoker & $63(18.3)$ & $17(11.4)$ & 0.0053 \\
\hline Ex-smoker & $261(75.9)$ & $112(75.2)$ & \\
\hline Never-smoker & $20(5.8)$ & $20(13.4)$ & \\
\hline Pack-years & $34.2 \pm 15.5$ & $30.6 \pm 16.8$ & 0.0134 \\
\hline \multicolumn{4}{|l|}{ Comorbidities } \\
\hline Gastrointestinal & $149(41.7)$ & $105(56.2)$ & 0.0014 \\
\hline Asthma & $65(18.2)$ & $50(26.7)$ & 0.0206 \\
\hline Hyperuricaemia & $52(14.6)$ & $38(20.3)$ & 0.0862 \\
\hline Hyperlipidaemia & $150(42.0)$ & $88(47.1)$ & 0.2602 \\
\hline Osteoporosis & $43(12.0)$ & $32(17.1)$ & 0.1035 \\
\hline Arterial hypertension & $187(52.4)$ & $100(53.5)$ & 0.8080 \\
\hline Coronary artery disease & $49(13.7)$ & $25(13.4)$ & 0.9083 \\
\hline Diabetes & $40(19.7)$ & $26(13.8)$ & 0.2812 \\
\hline \multicolumn{4}{|l|}{ Medication } \\
\hline OCS & $22(5.9)$ & $31(16.4)$ & $<0.0001$ \\
\hline ICS & $217(57.9)$ & $120(63.5)$ & 0.1985 \\
\hline $\mathrm{FEV}_{1}$ (\% predicted) & $61 \pm 22.4$ & $58.2 \pm 21.7$ & 0.1550 \\
\hline $\mathrm{FEV}_{1} / \mathrm{FVC}$ & $0.55 \pm 0.14$ & $0.57 \pm 0.14$ & 0.1678 \\
\hline BMI $\mathrm{kg} \cdot \mathrm{m}^{-2}$ & $26.9 \pm 4.8$ & $27.3 \pm 5.5$ & 0.3224 \\
\hline GOLD 1 & $40(10.7)$ & $13(7.0)$ & 0.1972 \\
\hline GOLD 2 & $141(37.8)$ & $63(33.9)$ & \\
\hline GOLD 3 & $116(31.1)$ & $62(33.3)$ & \\
\hline GOLD 4 & $20(5.4)$ & $14(7.5)$ & \\
\hline GOLD 0 & $56(15.0)$ & $34(18.3)$ & \\
\hline GOLD A & $55(14.8)$ & $24(12.9)$ & 0.7680 \\
\hline GOLD B & $210(56.6)$ & $105(56.5)$ & \\
\hline GOLD C & $3(0.8)$ & $3(1.6)$ & \\
\hline GOLD D & $103(27.8)$ & $54(29.0)$ & \\
\hline PHQ-9 & $5.6 \pm 4.3$ & $6 \pm 4.3$ & 0.3800 \\
\hline CAT score & $17.1 \pm 6.9$ & $17.9 \pm 7.3$ & 0.1786 \\
\hline mMRC & $1.5 \pm 0.9$ & $1.5 \pm 0.9$ & 0.5769 \\
\hline \multicolumn{4}{|c|}{$\begin{array}{l}\text { Data expressed as } n(\%) \text { and mean } \pm \text { SD unless otherwise stated. Baseline characteristics of the study population } \\
\text { as well as the excluded patients. Comparisons between groups were performed by chi-squared statistics for } \\
\text { categorical variables and by ANOVA for continuous variables. The level of significance was set at p<0.05. } \\
\text { OCS: oral corticosteroids; ICS: inhaled corticosteroids; FEV }{ }_{1} \text { : forced expiratory volume in } 1 \mathrm{~s} \text {; FVC: forced vital } \\
\text { capacity; BMI: body mass index; GOLD: Global Initiative for Chronic Obstructive Lung Disease; PHQ-9: Patient } \\
\text { Health Questionnaire Depression Scale 9; CAT: COPD Assessment Test; mMRC: Modified Medical Research } \\
\text { Council. }\end{array}$} \\
\hline
\end{tabular}

Information on infection control measures

Figure 2e shows the results regarding the source of information on infection control measures. Whereas the percentage of patients relying on public media has been high throughout and only slightly decreased in COVID2, the reliance on general practitioners, pneumologists and pneumological clinics as sources of information increased from COVID 1 to COVID2.

\section{Course of COPD}

Figure 3a shows symptoms and clinical state. Overall, from COVID1 to COVID2 patients reported increased dyspnoea (cough and deteriorations in form of exacerbations), as well as a deterioration of their general clinical state. The number of patients who reported no increase in dyspnoea decreased from $56 \%$ to $46 \%$, and the number of patients who reported low cough complaints decreased from $64 \%$ to $56 \%$. Furthermore, the proportion of patients reporting a stable general health status decreased from $76 \%$ to $71 \%$. 


\begin{tabular}{|c|c|c|c|c|c|c|c|}
\hline & COSYCONET (1) & COVID 1 (2) & COVID 2 (3) & $\begin{array}{c}\text { Mean change } \\
1 \text { to } 2\end{array}$ & $\begin{array}{c}\text { Mean change } \\
2 \text { to } 3\end{array}$ & $\begin{array}{c}\text { p-value } \\
1 \text { versus } 2 \\
\text { "before/during" }\end{array}$ & $\begin{array}{c}\text { p-value } \\
2 \text { versus } 3 \\
\text { "during pandemic" }\end{array}$ \\
\hline CAT item & $17.1 \pm 6.9$ & $18.0 \pm 7.5$ & $18.5 \pm 7.6$ & +0.90 & +0.05 & 0.0003 & 0.0417 \\
\hline (1) Cough & $2.16 \pm 1.10$ & $2.25 \pm 1.17$ & $2.34 \pm 1.17$ & +0.09 & +0.09 & 0.1272 & 0.0879 \\
\hline (2) Sputum & $2.17 \pm 1.23$ & $2.19 \pm 1.33$ & $2.23 \pm 1.31$ & +0.02 & +0.04 & 0.7937 & 0.5208 \\
\hline (3) Chest tightness & $1.74 \pm 1.24$ & $1.68 \pm 1.35$ & $1.85 \pm 1.39$ & -0.06 & +0.17 & $<0.0001$ & 0.0074 \\
\hline (4) Dyspnoea/stairs & $3.59 \pm 1.27$ & $3.77 \pm 1.30$ & $3.78 \pm 1.36$ & +0.18 & +0.01 & $<0.0001$ & 0.6409 \\
\hline (5) Limitations/home activities & $2.10 \pm 1.47$ & $2.35 \pm 1.59$ & $2.44 \pm 1.59$ & +0.25 & +0.09 & 0.0555 & 0.1212 \\
\hline (6) Confidence leaving home & $0.80 \pm 1.17$ & $1.16 \pm 1.52$ & $1.14 \pm 1.47$ & +0.36 & -0.02 & $<0.0001$ & 0.8585 \\
\hline (7) Sleep & $2.00 \pm 1.43$ & $2.03 \pm 1.55$ & $2.12 \pm 1.51$ & +0.03 & +0.09 & 0.1321 & 0.2743 \\
\hline (8) Energy & $2.40 \pm 1.17$ & $2.59 \pm 1.30$ & $2.64 \pm 1.29$ & +0.19 & +0.05 & $<0.0001$ & 0.4507 \\
\hline mMRC & $1.47 \pm 0.91$ & $1.62 \pm 0.97$ & $1.70 \pm 1.01$ & +0.15 & +0.08 & $<0.0001$ & 0.0673 \\
\hline PHQ-9 & $5.63 \pm 4.27$ & $5.98 \pm 4.92$ & $6.16 \pm 4.65$ & +0.35 & +0.18 & 0.0863 & 0.2318 \\
\hline Healthy & $170(54.8)$ & $173(46.4)$ & $161(45.1)$ & & & $<0.0001$ & $<0.0001$ \\
\hline Clinically unremarkable & $142(38.3)$ & $132(35.4)$ & $122(34.2)$ & & & & \\
\hline Mild depression & $43(11.6)$ & $40(10.7)$ & $52(14.6)$ & & & & \\
\hline Moderate depression & $11(3.0)$ & $19(5.1)$ & $18(5.0)$ & & & & \\
\hline Severe depression & $5(1.4)$ & $9(2.4)$ & $4(1.1)$ & & & & \\
\hline
\end{tabular}

Data expressed as $n(\%)$ and mean \pm SD unless otherwise stated. Comparison of changes of the single CAT items, the PHQ-9 as well as the mMRC between the pre-COVID COSYCONET visit and COVID1, as well as the comparison between the two COVID surveys during the pandemic. The level of significance was set at $p<0.05$. The comparisons between groups were performed using pairwise t-test for the metric variables. Additionally, the PHQ-9 categories were compared using Wilcoxon signed-rank tests for dependent samples.

Physical activity

Figure 3b shows physical activity as self-reported in COVID1 and COVID2. Reductions of general physical activity were attributable to the restrictions on free movement and scheduled physiotherapy. However, there were only minor changes between COVID1 and COVID2 with an increase of 3\% of patients reporting low general physical capacity. Regardless, general physical capacity was generally considered lower by patients.

\section{Additional changes in the COVID3 assessment}

When extending the scope of assessments to COVID3, we observed stable results for most of the items presented above, except for general physical capacity and physical condition, which significantly deteriorated, as reflected in increased cough and dyspnoea (see figure 4).

When repeating the comparison of COVID1 and COVID2 responses for this subpopulation, the results were compatible with those initially reported, especially for higher adherence to hand disinfection, less avoidance of contact to practices and increasing level of accessibility to general practitioners and pulmonologists for COVID2 compared to the COVID1 answers.

\section{Relationship between pre-COVID state and COVID1 results}

Responses to COVID1 were in a first step compared with the last COSYCONET visit and in a second step with the responses to COVID2 (table 2). The comparison between pre-COVID and COVID 1 showed a slight increase of total CAT score $(\mathrm{p}<0.05)$, corresponding to increases in CAT items 4,6 and 8 (dyspnoea/stairs, confidence leaving home and energy), and a decrease in CAT item 3 (chest tightness). Accordingly, mMRC was higher and the relative frequency of elevated PHQ-9 scores (upper three categories) was higher, as reflected in higher rates of moderate and severe depression (table 2). Further information on the comparison of physical characteristics and specific COVID survey items, as well as their relationship to the annual decline of lung function, can be found in the supplementary material.

\section{Discussion}

In the present study, we assessed the impact of infection control measures against COVID-19 on medical treatment, protective behaviour, symptoms, exacerbations and clinical state of COPD patients. We also analysed whether the results depended on the individual clinical history, which was possible as the study was performed within the long-term COSYCONET follow-up framework. Overall, patients showed high adherence to protective measures and over time increasingly relied on information from medical 

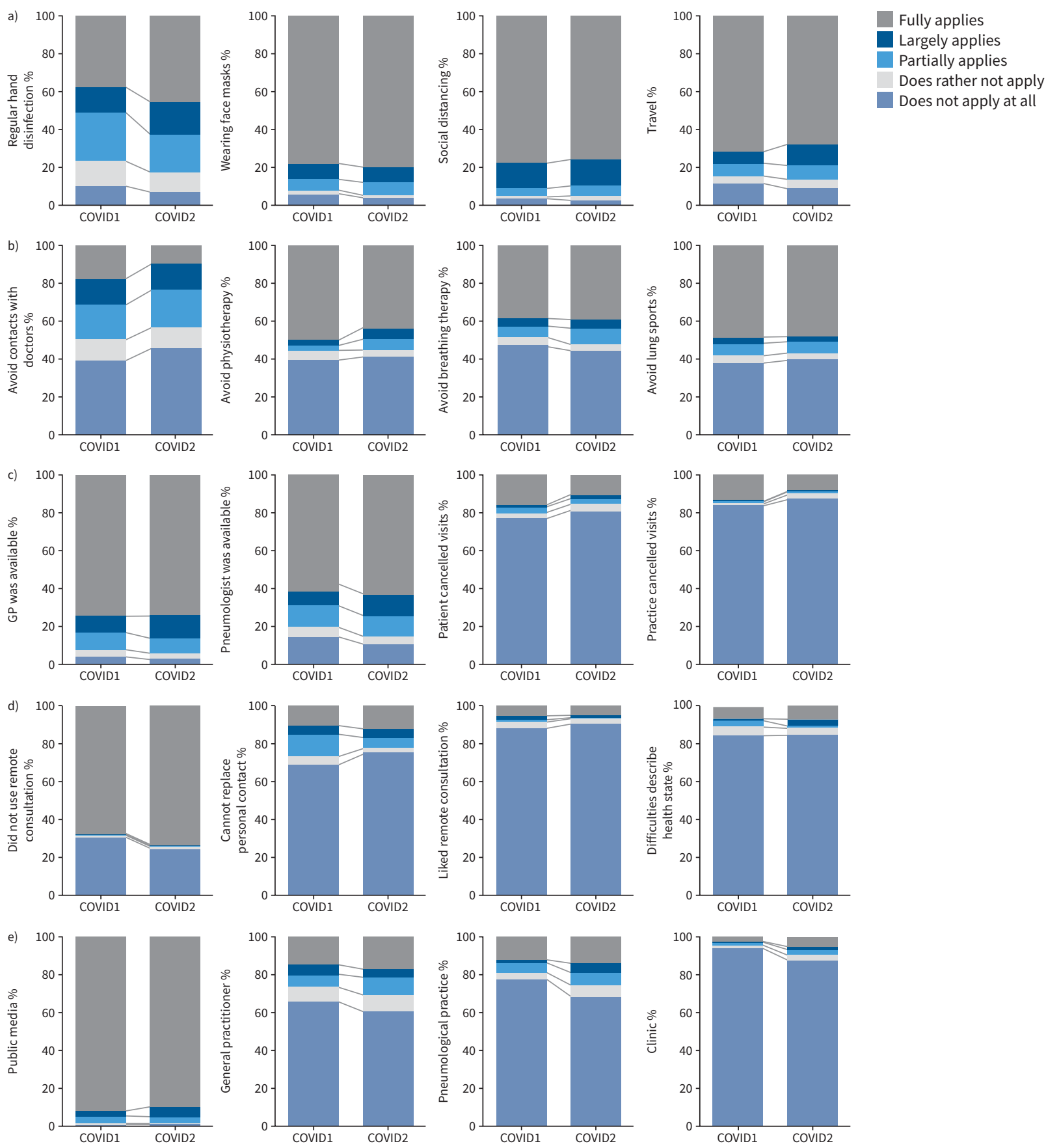

FIGURE 2 Repeated surveys regarding patients' behaviour. Results of COVID1 and COVID 2 regarding individual measures of hygiene (a), disease-specific behaviour (b), access to medical care (c), video or telephone consultation (d) and information on infection control measures (e). Answers range from "does not apply at all" to "fully applies".

professionals. Generally, adherence was higher in women, but interestingly, adherence was lower in patients with higher education. Symptoms slightly increased and self-estimated physical capacity decreased over time. The negative impact of infection control measures on the clinical state was stronger in patients with a history of frequent exacerbations, while the adherence of such patients to the measures partially even decreased. Similar results were observed for patients with comorbidities described as risk factors for COVID-19, such as diabetes, obesity or coronary artery disease. Although changes were small, these findings indicate deteriorations of self-reported clinical state in patients with COPD despite an increasing adherence to protective measures against COVID-19. 

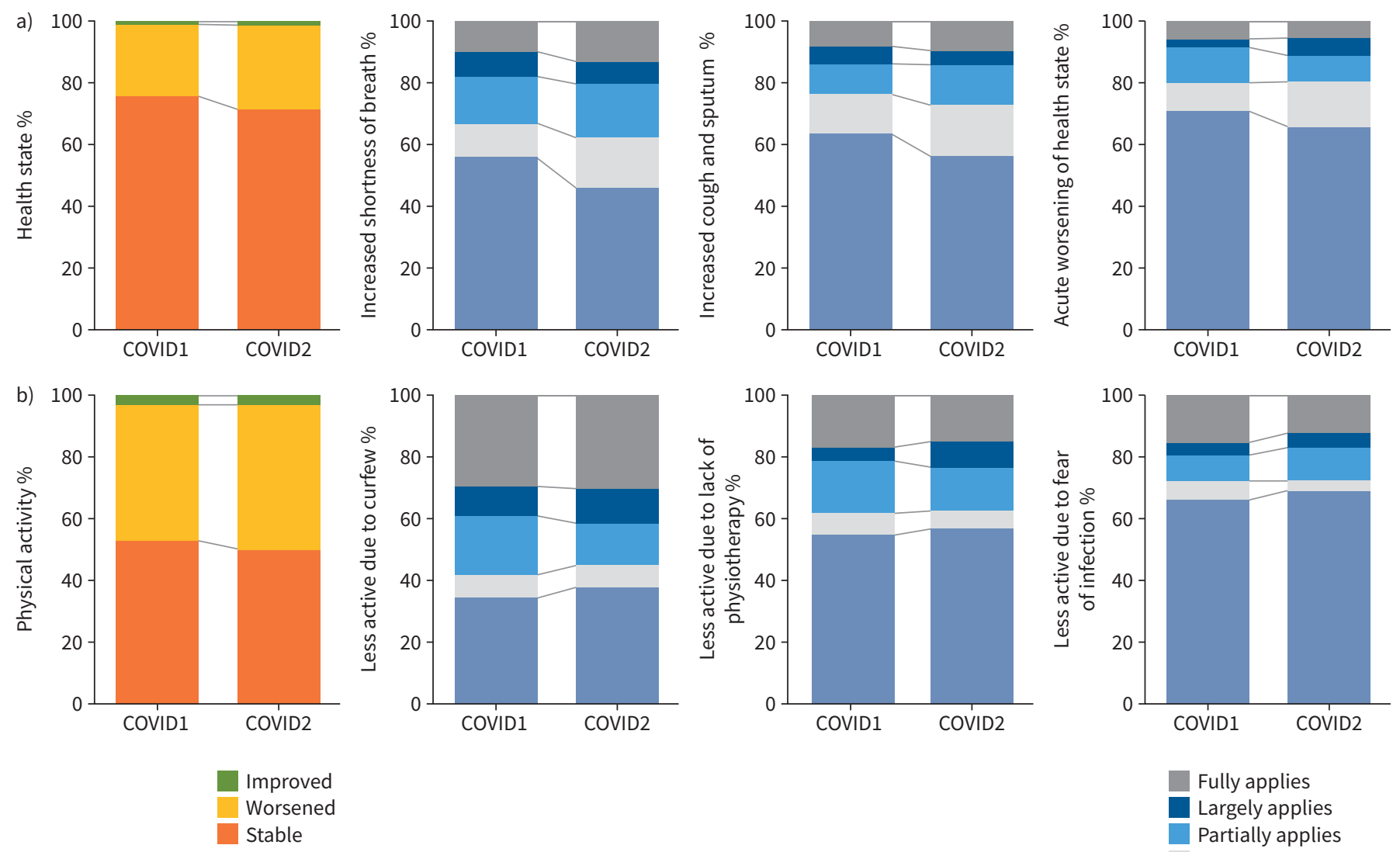

Improved

Stable

FIGURE 3 Repeated surveys regarding health status and physical activity. Results of COVID1 and COVID 2 regarding the course of COPD: a) health status and b) physical activity. Answers range from "does not apply at all" to "fully applies".

In our study cohort, only a small fraction of patients was amenable to tele-consultations. For the vast majority, personal contact with physicians was still important. This was reflected in the tendency over the course of the study to obtain more information from physicians and increase face to face contact with them. This suggests that irrespective of the advantages of remote care, patients rely on the personal contact with physicians at regular intervals, especially as there are differences between physicians and patients regarding the perception and evaluation of clinical state [14]. On the other hand, in our study COPD patients with high symptom burden and exacerbation risk reported avoiding contact with physicians in favour of tele-consultation. This indicates that remote care should be tailored to the specific needs of a certain group of patients.

While several studies reported that the number of exacerbations leading to hospitalisation decreased during the COVID pandemic [15, 16], two studies showed an increase or at least frequent exacerbations during the first phase of COVID-19 in the UK and Spain [4, 17]. Our data showed an increase in symptoms indicative of mild exacerbations. This may result from different definitions. Also, stronger protection against infectious agents may have prevented moderate and severe exacerbations, whereas the reduction in medical care led to a slight deterioration over time. Despite reductions in medical care, patients did not report increased impediments to access respiratory medication and denied a decrease in the quality of respiratory care (see supplementary figure S1). The numbers of appointments cancelled by either physicians or patients also decreased over time, which indicates that both sides got accustomed to the new situation. Regardless, the self-reported physical capacity of the patients decreased, in accordance with objective measurements [18].

The assumption that the protective measures against COVID-19 were effective is in line with the observation that only five of 564 patients became infected with COVID-19. Considering that severe exacerbations are known to have a major impact on the course of the disease [3, 19], it could be argued that in the future COPD patients might benefit from some of the protective measures established during the 

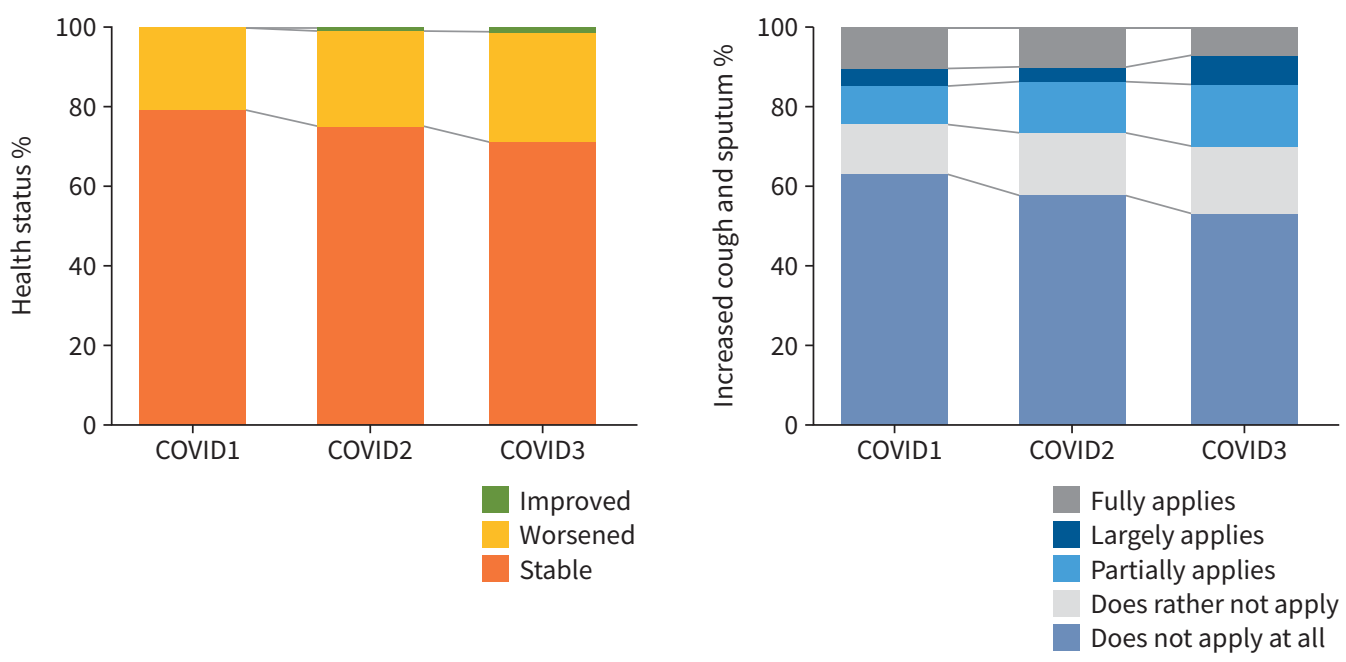

FIGURE 4 Additional changes in the COVID3 assessment. Answers for "increased cough and sputum production" range from "does not apply at all" to "fully applies".

pandemic, such as a careful selection of contacts and the wearing of face masks under specific conditions. While a previous analysis of COSYCONET data showed an association between better lung function and higher educational status [13], the present study found lower levels of adherence to infection control measures among patients with higher education. This should be considered when advising patients on infection control measures.

\section{Limitations}

Owing to the low number of patients with COVID-19, our data does not allow to draw conclusions on the impact of this disease in COPD. By contrast, the data reflects the impact of protective measures against COVID-19 on these patients, which is particularly relevant for the majority of our study population who did not suffer from COVID-19. Although the initial response rate to the questionnaires of 95\% was rather high, the number of responses decreased with each iteration of the questionnaire, especially between COVID2 and COVID3 (see table 1). Except for a higher prevalence of asthma, gastrointestinal disorders and the use of oral corticosteroids, the characteristics were very similar between the dropouts and the patients having at least the first two assessments. Regarding the occurrence of increased symptoms and deteriorations compatible with mild exacerbations, we had to rely on patients' answers as we naturally could not include functional measurements or physical examinations that were avoided by a significant number of patients. The assessment of single CAT items has not been validated so far. Nevertheless, we analysed the single CAT items as a proxy of COPD-specific symptoms, which was shown to be an effective approach in previous COSYCONET investigations [20]. This also allowed a direct comparison of the pre-COVID and COVID era.

\section{Conclusion}

When monitoring the potentially disease-relevant behaviour and clinical state of well-characterised COPD patients from May 2020 to the beginning of 2021 through the responses to three repeated questionnaires, we observed slight deteriorations of clinical state and self-reported exercise capacity despite high and partially increasing adherence to the protective measures and at the same time decreasing limitations in the access to medical care. According to most patients, the personal treatment by physicians could not be adequately substituted by remote care. However, women and COPD patients with high symptom burden and exacerbation risk reported an increased avoidance of personal contacts with physicians, while all patients did not favour tele-consultation. Therefore, the results suggest that in particular women and GOLD $\mathrm{D}$ patients should be actively contacted by the physician for the diagnosis of deteriorations.

Acknowledgements: We are grateful to the COSYCONET study group and study centres that contributed in patient recruitment and data collection, as well as to all patients participating in this study.

COSYCONET study group: Andreas, Stefan (Lungenfachklinik, Immenhausen); Bals, Robert Universitätsklinikum des Saarlandes); Behr, Jürgen and Kahnert, Kathrin (Klinikum der Ludwig-Maximilians-Universität München); Bahmer, 
Thomas (Universitätsklinikum Schleswig-Holstein) and Bewig, Burkhard (Städtisches Krankenhaus Kiel); Ewert, Ralf and Stubbe, Beate (Universitätsmedizin Greifswald); Ficker, Joachim H. (Klinikum Nürnberg, Paracelsus Medizinische Privatuniversität Nürnberg); Grohé, Christian (Ev. Lungenklinik Berlin); Held, Matthias (Klinikum Würzburg Mitte gGmbH, Standort Missioklinik); Behr, Jürgen and Henke, Markus (Asklepios Fachkliniken München-Gauting); Herth, Felix (Thoraxklinik Heidelberg $\mathrm{gGmbH}$ ); Kirsten, Anne-Marie and Watz, Henrik (Pneumologisches Forschungsinstitut an der Lungenclinic Grosshansdorf GmbH); Koczulla, Rembert (Schön Klinik Berchtesgadener Land); Kronsbein, Juliane (Berufsgenossenschaftliches Universitätsklinikum Bergmannsheil, Bochum); Kropf-Sanchen, Cornelia (Universitätsklinikum Ulm); Herzmann, Christian (Forschungszentrum Borstel); Pfeifer, Michael (Klinik Donaustauf); Randerath, Winfried J. (Wissenschaftliches Institut Bethanien e. V., Solingen); Seeger, Werner (Justus-Liebig-Universität Gießen); Studnicka, Michael (Uniklinikum Salzburg); Taube, Christian (Ruhrlandklinik gGmbH Essen); Timmermann, Hartmut (Hamburger Institut für Therapieforschung GmbH); Alter, Peter, Schmeck, Bernd and Vogelmeier, Claus (Universitätsklinikum Gießen und Marburg GmbH, Standort Marburg); Welte, Tobias (Medizinische Hochschule Hannover); Wirtz, Hubert (Universitätsklinikum Leipzig)

Study nurses: Doris Lehnert, Evangelische Lungenklinik Berlin; Birte Struck, Bergmannsheil Berufsgenossenschaftliches Universitätsklinikum Bochum; Lenka Krabbe, Medizinische-Klinik Borstel; Barbara Arikan, Julia Tobias, Klinik Donaustauf; Gina Spangel, Julia Teng, Ruhrlandklinik gGmbH Essen; Kornelia Speth, Universitätsklinikum Gießen; Jeanette Pieper, Universitätsmedizin Greifswald; Margret Gleiniger, Britta Markworth, Zaklina Hinz, Petra Hundack-Winter, Pneumologisches Forschungsinstitut Großhansdorf; Ellen Burmann, Hamburger Institut für Therapieforschung Hamburg; Katrin Wons, Sylvia Wagner Medizinische Hochschule Hannover; Ulrike Rieber, Beate Schaufler, Thoraxklinik am Universitätsklinikum Heidelberg; Martina Seibert, Universitätsklinikum des Saarlandes, Homburg/Saar; Katrin Schwedler, Lungenfachklinik Immenhausen; Sabine Michalewski, Sonja Rohweder, Universitätsklinikum Schleswig-Holstein, Campus Kiel; Patricia Berger, Universitätsklinikum Leipzig; Diana Schottel, Krankenhaus Lindenbrunn, Coppenbrügge; Manuel Klöser, Universitätsmedizin der Johannes Gutenberg-Universität Mainz; Vivien Janke, Universitätsklinikum Marburg; Rosalie Untsch, Asklepios Fachkliniken, München-Gauting; Jana Graf, Klinikum der Universität München; Anita Reichel, Klinikum Nürnberg; Gertraud Weiß, Erich Traugott, Barbara Ziss, Schön Klinik Berchtesgadener Land; Ilona Kietzmann, Wissenschaftliches Institut Bethanien für Pneumologie e.V, Solingen; Michaela Schrade-Illmann, Beate Polte, Universitätsklinikum Ulm; Cornelia Böckmann, Gudrun Hübner, Lena Sterk, Anne Wirz, Klinikum Würzburg Mitte gGmbH, Standort Missioklinik, Würzburg.

The COSYCONET framework is registered at www.clinicaltrials.gov with identifier number NCT01245933 [5]. The basic data are and available upon request. There is a detailed procedure for this at www.asconet.net. Specifically, the data can be obtained by submission of a proposal that is evaluated by the steering committee. All results to which the manuscript refers to are documented by the appropriate in the text, figures or tables.

Ethics approval and consent to participate: All assessments were approved by the central (Ethikkommission FB Medizin Marburg) and local (Bad Reichenhall (Ethikkommission bayerische Landesärztekammer); Berlin (Ethikkommission Ärztekammer Berlin); Bochum (Ethikkommission Medizinische Fakultät der RUB); Borstel (Ethikkommission Universität Lübeck); Coswig (Ethikkommission TU Dresden); Donaustauf (Ethikkommission Universitätsklinikum Regensburg); Essen (Ethikkommission Medizinische Fakultät Duisburg-Essen); Gießen (Ethikkommission Fachbereich Medizin); Greifswald (Ethikkommission Universitätsmedizin Greifswald); Großhansdorf (Ethikkommission Ärztekammer Schleswig-Holstein); Hamburg (Ethikkommission Ärztekammer Hamburg); MHH Hannover/Coppenbrügge (MHH Ethikkommission); Heidelberg Thorax/Uniklinik (Ethikkommission Universität Heidelberg); Homburg (Ethikkommission Saarbrücken); Immenhausen (Ethikkommission Landesärztekammer Hessen); Kiel (Ethikkommission Christian-Albrechts-Universität zu Kiel); Leipzig (Ethikkommission Universität Leipzig); Löwenstein (Ethikkommission Landesärztekammer Baden-Württemberg); Mainz (Ethikkommission Landesärztekammer Rheinland-Pfalz); München LMU/Gauting (Ethikkommission Klinikum Universität München); Nürnberg (Ethikkommission Friedrich-Alexander-Universität Erlangen Nürnberg); Rostock (Ethikkommission Universität Rostock); Berchtesgadener Land (Ethikkommission); Schmallenberg (Ethikkommission Ärztekammer Westfalen-Lippe); Solingen (Ethikkommission Universität Witten-Herdecke); Ulm (Ethikkommission Universität UIm); Würzburg (Ethikkommission Universität Würzburg)) ethical committees, and written informed consent was obtained from all patients. The additional examination of patients with the COVID questionnaire was also approved by the Ethics Committee (COSYCONET study, Amendment 11, AZ 200/09).

Consent for publication: Within the ethical approval the participants of the study gave their consent to publish the data collected during the study period.

Conflict of interest: K. Kahnert has nothing to disclose. J.I. Lutter has nothing to disclose. T. Welte reports grants from German Ministry of Research and Education during the conduct of the study. P. Alter has nothing to disclose. J. Behr has nothing to disclose. F. Herth has nothing to disclose. H-U. Kauczor reports other support from Bayer 
and Siemens during the conduct of the study; and personal fees and other support from Philips, and personal fees from AstraZeneca, Merck Sharp Dohme and Boehringer Ingelheim, outside the submitted work. S. Söhler has nothing to disclose. M. Pfeifer has nothing to disclose. H. Watz has nothing to disclose. C.F. Vogelmeier reports grants, personal fees and other support from AstraZeneca, Boehringer Ingelheim, GlaxoSmithKline and Novartis, personal fees and other support from CSL Behring, Chiesi, Grifols and Nuvaira, and personal fees from Berlin Chemie, outside the submitted work. R. Bals reports grants from BMBF during the conduct of the study; COSYCONET is supported by the German Centre for Lung Research (DZL) grant number 82DZLI05A2 (COSYCONET), German Federal Ministry of Education and Research (BMBF) grant number 01GI0881, and is furthermore supported by unrestricted grants from AstraZeneca $\mathrm{GmbH}$, Boehringer Ingelheim Pharma GmbH \& Co. KG, GlaxoSmithKline GmbH\&Co. KG, Grifols Deutschland $\mathrm{GmbH}$, and Novartis Deutschland $\mathrm{GmbH}$; and grants and personal fees from AstraZeneca and Boehringer Ingelheim, personal fees from GlaxoSmithKline and Grifols, grants and personal fees from Novartis, personal fees from CSL Behring, grants from BMBF Competence Network Asthma and COPD (ASCONET), Sander Stiftung, Schwiete Stiftung, Krebshilfe and Mukoviszidose eV, outside the submitted work; and a leadership or fiduciary role in the Alpha-1-Center. R.A. Jörres has nothing to disclose. F.C. Trudzinski has nothing to disclose.

Support statement: This investigation was supported by Chiesi $\mathrm{GmbH}$. The study was conducted within the COSYCONET framework. COSYCONET is supported by German Centre for Lung Research (DZL) grant number 82DZLI05A2 (COSYCONET), BMBF grant number 01GI088,1 and is furthermore supported by unrestricted grants from AstraZeneca GmbH, Boehringer Ingelheim Pharma GmbH \& Co. KG, GlaxoSmithKline GmbH\&Co. KG, Grifols Deutschland $\mathrm{GmbH}$, and Novartis Deutschland $\mathrm{GmbH}$. Funding information for this article has been deposited with the Crossref Funder Registry.

\section{References}

1 Lippi G, Henry BM. Chronic obstructive pulmonary disease is associated with severe coronavirus disease 2019 (COVID-19). Respir Med 2020; 167: 105941.

2 Alqahtani JS, Oyelade T, Aldhahir AM, et al. Prevalence, severity and mortality associated with COPD and smoking in patients with COVID-19: a rapid systematic review and meta-analysis. PLOS ONE 2020; 15: e0233147.

3 Halpin DMG, Criner GJ, Papi A, et al. Global Initiative for the Diagnosis, Management, and Prevention of Chronic Obstructive Lung Disease. The 2020 GOLD Science Committee Report on COVID-19 and Chronic Obstructive Pulmonary Disease. Am J Respir Crit Care Med 2021; 203: 24-36.

4 Pleguezuelos E, Del Carmen A, Moreno E, et al. The experience of COPD patients in lockdown due to the COVID-19 pandemic. Int J Chron Obstruct Pulmon Dis 2020; 15: 2621-2627.

5 Karch A, Vogelmeier C, Welte T, et al. The German COPD cohort COSYCONET: aims, methods and descriptive analysis of the study population at baseline. Respir Med 2016; 114: 27-37.

6 Celli BR, Decramer M, Wedzicha JA, et al. An Official American Thoracic Society/European Respiratory Society Statement: research questions in chronic obstructive pulmonary disease. Am J Respir Crit Care Med 2015; 191 e4-e27.

7 Criee CP, Baur X, Berdel D, et al. [Standardization of spirometry: 2015 update. Published by German Atemwegsliga, German Respiratory Society and German Society of Occupational and Environmental Medicine]. Pneumologie 2015; 69: 147-164.

8 Stanojevic S, Graham BL, Cooper BG, et al. Official ERS technical standards: Global Lung Function Initiative reference values for the carbon monoxide transfer factor for Caucasians. Eur Respir J 2017; 50: 1700010.

9 Quanjer PH, Stanojevic S, Cole TJ, et al. Multi-ethnic reference values for spirometry for the 3-95-yr age range: the global lung function 2012 equations. Eur Respir J 2012; 40: 1324-1343.

10 Jones PW, Harding G, Berry P, et al. Development and first validation of the COPD Assessment Test. Eur Respir J 2009; 34: 648-654.

11 Mahler DA, Wells CK. Evaluation of clinical methods for rating dyspnea. Chest 1988; 93: 580-586.

12 Kroenke K, Spitzer RL, Williams JB. The PHQ-9: validity of a brief depression severity measure. J Gen Intern Med 2001; 16: 606-613.

13 Lutter JI, Jorres RA, Welte T, et al. Impact of education on COPD severity and all-cause mortality in lifetime never-smokers and longtime ex-smokers: results of the COSYCONET Cohort. Int J Chron Obstruct Pulmon Dis 2020; 15: 2787-2798.

14 Wu F, Burt J, Chowdhury T, et al. Specialty COPD care during COVID-19: patient and clinician perspectives on remote delivery. BMJ Open Respir Res 2021; 8: e000817.

15 Tan JY, Conceicao EP, Wee LE, et al. COVID-19 public health measures: a reduction in hospital admissions for COPD exacerbations. Thorax 2021; 76: 512-513.

16 Sykes DL, Faruqi S, Holdsworth L, et al. Impact of COVID-19 on COPD and asthma admissions, and the pandemic from a patient's perspective. ERJ Open Res 2021; 7: 00822-2020. 
17 McAuley H, Hadley K, Elneima O, et al. COPD in the time of COVID-19: an analysis of acute exacerbations and reported behavioural changes in patients with COPD. ERJ Open Res 2021; 7: 00718-2020.

18 Hume E, Armstrong M, Manifield J, et al. Impact of COVID-19 shielding on physical activity and quality of life in patients with COPD. Breathe (Sheff) 2020; 16: 200231.

19 Vogelmeier CF, Criner GJ, Martinez FJ, et al. Global strategy for the diagnosis, management, and prevention of chronic obstructive lung disease 2017 report: GOLD executive summary. Eur Respir J 2017; 49: 1700214.

20 Marietta von Siemens S, Alter P, Lutter JI, et al. CAT score single item analysis in patients with COPD: results from COSYCONET. Respir Med 2019; 159: 105810. 